\title{
Confinement Factors and Gain in Optical Amplifiers
}

\author{
T. D. Visser, H. Blok, Member, IEEE, B. Demeulenaere, Member, IEEE, and D. Lenstra, Member, IEEE
}

\begin{abstract}
A new identity is derived which relates the gain and the field distribution (or confinement factor) in a dielectric waveguide with complex refractive indices. This identity is valid for any guided mode of waveguides with an arbitrary cross section. It provides a new check of the accuracy of mode solvers. Also, it can be used in a variational approach to predict the gain or loss of a guided mode based on knowledge of confinement factors. It is shown that a previous analysis that is often used, is not correct. In addition, approximate expressions for the gain in slab waveguides are presented.
\end{abstract}

Index Terms - Polarization, semiconductor device modeling, semiconductor laser amplifiers, traveling-wave amplifiers, waveguide theory.

\section{INTRODUCTION}

$\mathbf{T}$ HE SUPPRESSION of polarization sensitivity in semiconductor traveling-wave amplifiers (TWA's) has recently received a lot of attention [1], [2], [3]. In order to study this, a so-called modal analysis of the device is needed. In recent papers, the authors have investigated guided modes in planar dielectric waveguides with gain and losses [4]-[7]. In semiconductor TWA's, the material gain in the active region due to carrier injection introduces an additional positive imaginary part to the index of refraction [8]. Likewise, losses in the cladding layers are represented by a negative imaginary part of the refractive index. In the present paper we study the relation between optical confinement and modal gain in waveguides of arbitrary cross section.

For active linear waveguides (i.e., with complex-valued indices of refraction) there exist two kinds of methods to obtain the modal gain. The first ones are the indirect approximate methods, such as the effective index method [9] or variational methods [10], which use results for the corresponding passive waveguide (with real-valued refractive indices), to predict the modal gain of the active configuration. Since the imaginary part of the refractive index in the active waveguide is typically small compared to the real part, it can be considered as a kind

Manuscript received April 14, 1997; revised June 11, 1997. This work was supported by the Technology Foundation (STW). The work of B Demeulenaere was supported by a doctoral fellowship from the National Fund for Scientific Research of Belgium (NFWO). The work of H. Blok was supported by a grant from the Fund for Science, Technology and Research, Schlumberger Limited, New York.

T. D. Visser is with the Department of Physics and Astronomy, Free University, $1081 \mathrm{HV}$ Amsterdam, The Netherlands. He is also with the Laboratory of Electromagnetic Research, Department of Electrical Engineering, Delft University of Technology, 2600 GA Delft, The Netherlands.

$\mathrm{H}$. Blok is with the Laboratory of Electromagnetic Research, Department of Electrical Engineering, Delft University of Technology, 2600 GA Delft, The Netherlands.

B. Demeulenaere is with the Department of Information Technology, University of Gent, 9000 Gent, Belgium.

D. Lenstra is with the Department of Physics and Astronomy, Free University, 1081 HV Amsterdam, The Netherlands.

Publisher Item Identifier S 0018-9197(97)07084-X. of perturbation. That is, one can reasonably assume that the transverse field distribution in the active waveguide is very similar to that in the passive waveguide. The calculated field distribution and the ensueing confinement or filling factors for the passive waveguide can then be used to obtain an estimate of the modal gain of the active waveguide. An often used approximation for planar waveguides is

$$
g_{\bmod } \approx \sum_{i} \Gamma_{i}^{S_{z}} g_{i}
$$

where $g_{\text {mod }}$ is the modal gain, and $g_{i}$ is the plane wave bulk material gain or absorption of the $i$ th layer. The sum is over all layers that make up the waveguide. The confinement factor $\Gamma_{i}^{S_{z}}$ is defined as

$$
\Gamma_{i}^{S_{z}}=\frac{\int_{i}\left|\left\langle S_{z}(x, y)\right\rangle\right| d x d y}{\int_{-\infty}^{\infty}\left|\left\langle S_{z}(x, y)\right\rangle\right| d x d y}
$$

with $\left\langle S_{z}(x, y)\right\rangle$ the time-averaged $z$-component of the Poynting vector, and with the integral in the numerator over the $i$ th layer. Often the bulk material gain in the active layer is much larger than the bulk absorption in the cladding layers. In that case it suffices to use a single confinement factor, namely that of the active layer. Approximation (1) was derived by Adams under the assumption of weak guiding [11]. In that case, it suffices to use a scalar theory rather than a vector theory. An alternative derivation, also within the scalar regime, is given by Buus [8]. Although both authors clearly stress these limitations, (1) is used in very many papers, without questioning whether it is valid for the configuration under consideration. It has recently been pointed out for onedimensional (1-D) (i.e., planar) waveguides that expression (1) in certain cases of practical importance highly overestimates the gain for TM modes [5]. The explanation is that this approximation is based on the wave equation for TE modes. But TM modes satisfy another wave equation and hence the relation between modal gain and bulk gain is different (and more complex) for them [12]. ${ }^{1}$ Nevertheless, it is possible to use a different definition for the confinement factors so that (1) again gives a satisfactory prediction of the modal gain for both polarization states while still using the results of the passive waveguide [6], [7].

The second kind of method to calculate the modal gain is what we call the direct method. Contrary to the above mentioned indirect methods, these directly analyze the active waveguide with complex refractive indices. Examples are complex beam propagation methods [13], [14], and complex

\footnotetext{
${ }^{1}$ The fact that TM solutions satisfy a wave equation that differs from that for TE was also noticed by Huang et al. [12]. However, the authors make a mistake between their (8) and (9) by discarding the finite delta function contributions to the integral that they are considering.
} 
dispersion equation solvers [4]. For planar configurations, the accuracy of these complex mode solvers can be tested by checking if the obtained field distributions and effective permittivities satisfy certain identities [5]. It would be useful to have such an identity which can be used to check the accuracy of the calculated modal gain and the field distribution of twodimensional (2-D) waveguides. It is the aim of this paper to derive such an identity and to compare it with approximate expressions published earlier. We also show how this identity can be used to obtain approximate expressions for the gain of planar waveguides.

\section{GAIN AND CONFINEMENT IN 2-D WAVEGUIDES}

The theorem of Poynting or power theorem [15], [16] for monochromatic fields (i.e., with a time-dependence of $\exp [j \omega t])$ for a linear medium whose total electromagnetic response is characterized by a permittivity and a permeability reads

$$
\begin{aligned}
& \iint_{S}\left(\hat{\mathbf{E}} \times \hat{\mathbf{H}}^{*}\right) \cdot \mathbf{n} d S \\
& \quad=\iiint_{V}\left(j \omega \epsilon^{*} \hat{\mathbf{E}} \cdot \hat{\mathbf{E}}^{*}-j \omega \mu \hat{\mathbf{H}} \cdot \hat{\mathbf{H}}^{*}\right) d V .
\end{aligned}
$$

Here, * denotes complex conjugation and

$$
\begin{aligned}
\hat{\mathbf{E}} & =\text { electric field strength; } \\
\hat{\mathbf{H}} & =\text { magnetic field strength; } \\
\mathbf{n} & =\text { outward unit normal of the surface } S ; \\
V & =\text { volume that is bounded by the surface } S ; \\
j & =\text { imaginary unit; } \\
\omega & =\text { angular frequency; } \\
\epsilon & =\text { permittivity; } \\
\mu & =\text { permeability. }
\end{aligned}
$$

To include the effects of losses and gain, it is assumed that $\epsilon$ is a complex parameter, that is

$$
\epsilon=\epsilon^{\prime}-j \epsilon^{\prime \prime} \quad\left(\epsilon^{\prime}, \epsilon^{\prime \prime} \in \mathcal{R}\right) .
$$

The real part $\epsilon^{\prime}$ accounts for dispersion, whereas the imaginary part $\epsilon^{\prime \prime}$ is responsible for absorption or gain.

Consider now an active waveguide with arbitrary cross section, i.e., $\epsilon=\epsilon(x, y)$ (see Fig. 1). We study guided modes which propagate in the positive $z$-direction, i.e., electric and magnetic field distributions that are of the form

$$
\{\hat{\mathbf{E}}, \hat{\mathbf{H}}\}(x, y, z)=\{\tilde{\mathbf{E}}, \tilde{\mathbf{H}}\}(x, y) \exp \left[-j k_{z} z\right]
$$

where $k_{z}$, which is to be determined, is the complex propagation factor of a particular mode. That is,

$$
k_{z}=\beta-j \alpha \quad(\beta, \alpha \in \mathcal{R}) .
$$

The modal gain per length unit then equals

$$
g_{\mathrm{mod}}=-2 \alpha \text {. }
$$

Take $V$ in (3) to be a volume between the two planes $\mathcal{A}$ and $\mathcal{B}$ defined as $z=z_{0}$ and $z=z_{0}+\Delta z$, respectively (see Fig. 1). The other boundaries of $V$ are chosen far away from the active region, so that we may assume the fields to be exponentially

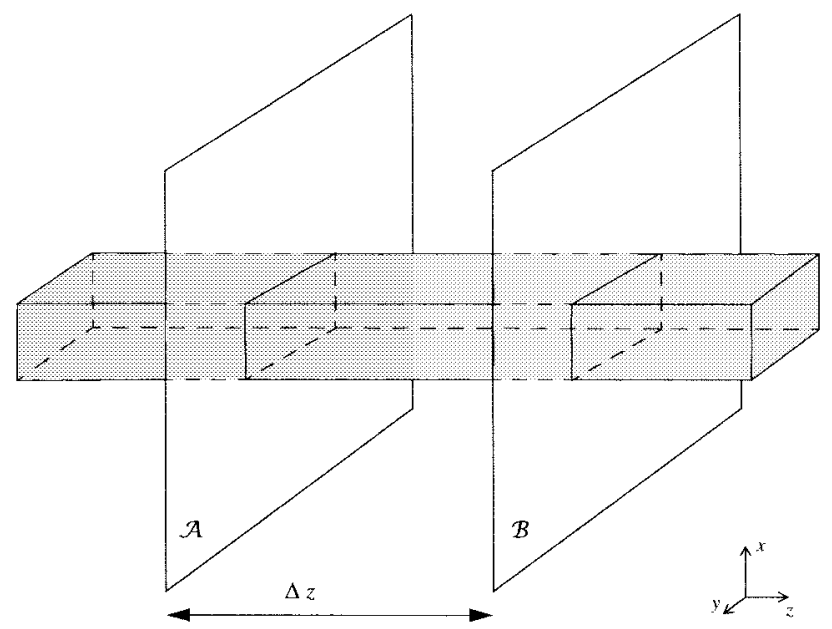

Fig. 1. An active semiconductor waveguide with an arbitrary cross section. The active region is shaded. The light propagates in the positive $z$-direction with complex propagation constant $k_{z}$. The planes $\mathcal{A}$ and $\mathcal{B}$ are both perpendicular to the $z$-axis.

decaying there. For the guided modes that we consider the total power flow takes place only in the $z$-direction. Hence, the surface integral in (3) reduces to

$$
-\int_{\mathcal{A}}\left(\hat{\mathbf{E}} \times \hat{\mathbf{H}}^{*}\right) \cdot \hat{\imath}_{z} d x d y+\int_{\mathcal{B}}\left(\hat{\mathbf{E}} \times \hat{\mathbf{H}}^{*}\right) \cdot \hat{\imath}_{z} d x d y
$$

where $\hat{\imath}_{z}=(0,0,1)$. For guided modes the factor $\left(\hat{\mathbf{E}} \times \hat{\mathbf{H}}^{*}\right)$ increases by a factor $\exp [-2 \alpha \Delta z]$ between plane $\mathcal{A}$ and plane $\mathcal{B}$ [see (7)]. So the surface integral of (3) equals

$$
\begin{aligned}
& \int_{S}\left(\hat{\mathbf{E}} \times \hat{\mathbf{H}}^{*}\right) \cdot \mathbf{n} d S \\
& \quad=(\exp [-2 \alpha \Delta z]-1) \int_{\mathcal{A}}\left(\tilde{\mathbf{E}} \times \tilde{\mathbf{H}}^{*}\right) \cdot \hat{\imath}_{z} d x d y .
\end{aligned}
$$

Since the configuration is independent of $z$, the volume integral in (3) can be written, with the help of (5), as

$$
\begin{aligned}
& j \omega \int_{V}\left(\epsilon^{*} \hat{\mathbf{E}} \cdot \hat{\mathbf{E}}^{*}-\mu \hat{\mathbf{H}} \cdot \hat{\mathbf{H}}^{*}\right) d V \\
& \quad=j \omega \int_{z_{0}}^{z_{0}+\Delta z} \exp [-2 \alpha z] d z \int_{\mathcal{A}}\left(\epsilon^{*}|\tilde{\mathbf{E}}|^{2}-\mu|\tilde{\mathbf{H}}|^{2}\right) d x d y \\
& \quad=\frac{-j \omega}{2 \alpha}(\exp [-2 \alpha \Delta z]-1) \int_{\mathcal{A}}\left(\epsilon^{*}|\tilde{\mathbf{E}}|^{2}-\mu|\tilde{\mathbf{H}}|^{2}\right) d x d y
\end{aligned}
$$

where we have set $z_{0}=0$. From now on we restrict ourselves to the important case of semiconductor waveguides, i.e. we set $\mu=\mu_{0}$, the permeability in vacuo. Substituting from (11) and (9) into (3) then gives us

$$
\begin{aligned}
- & 2 \alpha \int_{\mathcal{A}}\left(\tilde{\mathbf{E}} \times \tilde{\mathbf{H}}^{*}\right) \cdot \hat{\imath}_{z} d x d y \\
& =j \omega \int_{\mathcal{A}}\left(\epsilon^{\prime}|\tilde{\mathbf{E}}|^{2}-\mu|\tilde{\mathbf{H}}|^{2}\right) d x d y-\omega \int_{\mathcal{A}} \epsilon^{\prime \prime}|\tilde{\mathbf{E}}|^{2} d x d y .
\end{aligned}
$$

Next, we introduce the time-averaged power flow vector for a 
guided mode at a cross section $z$ as

$$
\langle\mathbf{S}(x, y, z)\rangle=\frac{1}{2} \operatorname{Re}\left\{\tilde{\mathbf{E}}(x, y) \times \tilde{\mathbf{H}}^{*}(x, y)\right\} e^{-2 \alpha z} .
$$

Combining the real parts of (12) and (13) gives

$$
\begin{aligned}
\alpha & =\frac{\omega}{4} \frac{\int_{\mathcal{A}} \epsilon^{\prime \prime}(x, y)|\hat{\mathbf{E}}(x, y)|^{2} d x d y}{\int_{\mathcal{A}}\langle\mathbf{S}(x, y, z)\rangle \cdot \hat{\imath}_{z} d x d y} \\
& =\frac{\omega}{4} \frac{\int_{\mathcal{A}} \epsilon^{\prime \prime}(x, y)|\tilde{\mathbf{E}}(x, y)|^{2} d x d y}{\frac{1}{2} \operatorname{Re} \int_{\mathcal{A}}\left(\tilde{\mathbf{E}}(x, y) \times \tilde{\mathbf{H}}(x, y)^{*}\right) \cdot \hat{\imath}_{z} d x d y}
\end{aligned}
$$

This identity, together with Eq. (7), states that the modal gain is proportional to the ratio of the integrated electric field energy density $|\hat{\mathbf{E}}(x, y)|^{2}$ of a guided mode weighed with $\epsilon^{\prime \prime}$, and the guided mode time-averaged power flow across any transverse cross section $\mathcal{A}$ of the active waveguide. It holds for any guided mode of any two-dimensional waveguide. Note that $\epsilon=\epsilon(x, y)$, i.e., (15) is valid for waveguides with an arbitrary refractive index profile.

One possible application lies in checking the accuracy of a complex 2-D mode solver which is used to analyze e.g., ridge or channel configurations. Also, the spurious solutions that some mode solvers generate will not satisfy (15) and can hence be identified. Another way of using it is in a variational approach as was sketched in Section I. The field and power flow distributions that are needed can then be approximated by the results from a mode solver that can analyze 2-D structures with real-valued refraction indices. Expression (15) then yields an approximation of the modal gain. In fact, if one uses the field distribution for the corresponding passive configuration, then (15) reduces to a perturbative expression suggested by Vassallo [17].

We note without proof that the expression (15) is stationary, so its use in a variational analysis is indeed justified. The proof is not given here since it is very similar to the one given by Harrington [10].

It should be noted that (15) implies that we cannot calculate the modal gain in terms of the fraction of the Poynting vector that is confined to the active layer. In other words, the term $\epsilon^{\prime \prime}|\hat{\mathbf{E}}(x, y)|^{2}$, which appears in the numerator is, generally speaking, not proportional to the power flow. The erroneous view that the modal gain is proportional to the confined fraction of the power flow is widely held. It was propagated in, e.g., [9], [18].

\section{GAIN VERSus CONFINEMENT RELATIONS FOR PLANAR WAVEGUIDES}

For planar waveguides, i.e., with the permittivity $\epsilon$ a piecewise constant function of $x$ only, the analysis can be carried out further. Assuming that the field is independent of $y$, it can be either TE or TM polarized. We first consider the former case. The only nonzero field components are then $\hat{E}_{y}, \hat{H}_{x}$ and $\hat{H}_{z}$. From (15), we have

$$
\alpha=\frac{\omega}{4} \frac{\int_{-\infty}^{\infty} \epsilon^{\prime \prime}\left|\tilde{E}_{y}\right|^{2} d x}{\frac{1}{2} \operatorname{Re}\left\{\int_{-\infty}^{\infty}-\tilde{E}_{y} \tilde{H}_{x}^{*} d x\right\}} .
$$

Using that

$$
\tilde{H}_{x}^{*}=\frac{-k_{z}^{*}}{\omega \mu_{0}} \tilde{E}_{y}^{*}
$$

gives

$$
\alpha=\frac{k_{0}^{2}}{2 \beta} \frac{\int_{-\infty}^{\infty} \epsilon_{r}^{\prime \prime}\left|\tilde{E}_{y}\right|^{2} d x}{\int_{-\infty}^{\infty}\left|\tilde{E}_{y}\right|^{2} d x}
$$

where we used that $k_{0}^{2}=\omega^{2} \mu_{0} \epsilon_{0}$ with $k_{0}$ the wavenumber in vасио and $\epsilon_{r}=\epsilon / \epsilon_{0}=\epsilon_{r}^{\prime}-j \epsilon_{r}^{\prime \prime}$. Let $n=n^{\prime}-j n^{\prime \prime}$ denote the index of refraction then, since $\epsilon_{r}=n^{2}$, we have $\epsilon_{r}^{\prime \prime}=2 n^{\prime} n^{\prime \prime}$. Let $n_{\text {eff }}=n_{\text {eff }}^{\prime}-j n_{\text {eff }}^{\prime \prime}$ be the effective index of a given mode. We know that [19]

$$
\max \left\{n_{\text {clad }}^{\prime}\right\}<n_{\text {eff }}^{\prime}<n_{\max }^{\prime}(x) \text {. }
$$

Here, $\max \left\{n_{\text {clad }}^{\prime}\right\}$ is the maximum value of $n^{\prime}$ of the cladding layers and $n_{\max }^{\prime}(x)$ is the maximum value of the real part of the bulk refractive index in the waveguide. Since $\beta=k_{0} n_{\text {eff }}^{\prime}$, (18) becomes

$$
\alpha=\frac{k_{0}}{n_{\text {eff }}^{\prime}} \frac{\int_{-\infty}^{\infty} n^{\prime} n^{\prime \prime}\left|\tilde{E}_{y}\right|^{2} d x}{\int_{-\infty}^{\infty}\left|\tilde{E}_{y}\right|^{2} d x}
$$

This rigorous expression for the gain holds for any TE mode in an arbitrary slab waveguide.

If we now assume that $n_{\text {eff }}^{\prime} \approx n^{\prime}$ (weak guiding approximation, cf. [8]), then for a slab waveguide with piecewise homogeneous layers, (20) reduces to the following approximation

$$
\alpha^{\mathrm{TE}} \approx k_{0} \sum_{i} n_{i}^{\prime \prime} \Gamma_{i}^{\mathrm{TE}}
$$

Here, the suffix $i$ indicates the layer, and the confinement factor $\Gamma_{i}^{\mathrm{TE}}$ is defined as

$$
\Gamma_{i}^{\mathrm{TE}}=\frac{\int_{\text {layer } i}\left|\tilde{E}_{y}\right|^{2} d x}{\int_{-\infty}^{\infty}\left|\tilde{E}_{y}\right|^{2} d x} .
$$

In case of a TM polarized field, the only nonzero field components are $\hat{H}_{y}, \hat{E}_{x}$, and $\hat{E}_{z}$. From (13) and (15), we have

$$
\frac{1}{2} \operatorname{Re}\left\{\tilde{\mathbf{E}} \times \tilde{\mathbf{H}}^{*}\right\} \cdot \hat{\imath}_{z}=\frac{1}{2} \operatorname{Re}\left\{\tilde{E}_{x} \tilde{H}_{y}^{*}\right\} .
$$

Also,

$$
\tilde{H}_{y}^{*}=\frac{\omega \epsilon^{*}}{k_{z}^{*}} \tilde{E}_{x}^{*}
$$

We eliminate $\tilde{H}_{y}$ because an expression for the TM gain in terms of that field component becomes rather convoluted, as it also contains its derivative. Substituting from (23) and (24) into (15) gives

$$
\alpha=\frac{\beta^{2}+\alpha^{2}}{2} \frac{\int_{-\infty}^{\infty} \epsilon^{\prime \prime}\left(\left|\tilde{E}_{x}\right|^{2}+\left|\tilde{E}_{z}\right|^{2}\right) d x}{\int_{-\infty}^{\infty}\left(\epsilon^{\prime} \beta+\epsilon^{\prime \prime} \alpha\right)\left|\tilde{E}_{x}\right|^{2} d x} .
$$

Using that in practice $\alpha^{2} \ll \beta^{2},\left|\epsilon^{\prime \prime} \alpha\right| \ll\left|\epsilon^{\prime} \beta\right|$ and $\left|\tilde{E}_{z}\right|^{2} \ll$ $\left|\tilde{E}_{x}\right|^{2}$ gives

$$
\alpha \approx \frac{\beta}{2} \frac{\int_{-\infty}^{\infty} \epsilon^{\prime \prime}\left|\tilde{E}_{x}\right|^{2} d x}{\int_{-\infty}^{\infty} \epsilon^{\prime}\left|\tilde{E}_{x}\right|^{2} d x}
$$




$$
=k_{0} n_{\mathrm{eff}} \frac{\int_{-\infty}^{\infty} n^{\prime} n^{\prime \prime}\left|\tilde{E}_{x}\right|^{2} d x}{\int_{-\infty}^{\infty}\left(n^{\prime 2}-n^{\prime 2}\right)\left|\tilde{E}_{x}\right|^{2} d x} .
$$

Neglecting the term with $n^{\prime 2}$ in the denominator and using again the assumption that $n_{\mathrm{eff}} \approx n^{\prime}$ gives for a slab waveguide

$$
\alpha^{\mathrm{TM}} \approx k_{0} \sum_{i} n_{i}^{\prime \prime} \Gamma_{i}^{\mathrm{TM}}
$$

where the confinement factor $\Gamma_{i}^{\mathrm{TM}}$ is defined as

$$
\Gamma_{i}^{\mathrm{TM}}=\frac{\int_{\text {layer } i}\left|\tilde{E}_{x}\right|^{2} d x}{\int_{-\infty}^{\infty}\left|\tilde{E}_{x}\right|^{2} d x} .
$$

So we find both for TE and TM polarization that with a suitable choice of the confinement factors an estimate for the modal gain can be obtained. Note that neither (21) nor (28) involves the power flow. This is in agreement with our earlier results [5], where the starting point was the wave equations for TE and TM rather than the complex power theorem. The present analysis is much simpler, and also suggests the use of $\tilde{E}_{x}$ rather than $\tilde{H}_{y}$ for the TM confinement factor. As remarked above, an expression for the TM gain in terms of $\tilde{H}_{y}$ is also possible but becomes very complex since it also contains the derivative of that field component.

As is discussed in [5], confinement factors that do involve the Poynting vector can give unacceptable results for TM polarized waves under typical circumstances. This point can be illustrated with the following numerical example. Consider a symmetric waveguide with $n_{\text {core }}=3.6+j 0.002$ and $n_{\text {clad }}=3.16-j 10^{-4}$. The core thickness was chosen as 0.1 $\mu \mathrm{m}$, the wavelength in vacuum was $\lambda_{0}=1.3 \mu \mathrm{m}$. The TM gain according to a mode solver [4] was $23.22 \mathrm{~cm}^{-1}$. This is in excellent agreement with (28), which predicts a value of $23.34 \mathrm{~cm}^{-1}$. However, it is in stark contrast to (1), which yields a value of $31.36 \mathrm{~cm}^{-1}$, more than $35 \%$ too high.

\section{CONCLUSION}

An identity was derived which relates the field distribution (or optical confinement) and the modal gain in semiconductor waveguides. The relation is valid for waveguides of arbitrary cross section. Also, this expression is stationary, so it can be used in a variational approach. Starting point was not (as in previous work) the wave equations, but the complex power theorem. It was shown that the gain of guided modes cannot be expressed, as is often thought, in terms of the fraction of the power flow that is confined to the active region.

A rigorous expression and two approximations for the gain in planar (i.e., slab) waveguides were obtained. It was found for that case too that these formulae are in terms of certain electric field components rather than the Poynting vector. It was shown that an approximation based on the power flow can become highly inaccurate, in contrast to the approximations that are suggested by the authors.

\section{REFERENCES}

[1] T. Saitoh and T. Mukai, "1.5 $\mu \mathrm{m}$ GaInAsP traveling-wave semiconductor laser amplifier," IEEE J. Quantum Electron., vol. 23, pp. 1010-1020, 1987.
[2] B. Mersali, "1.55 high gain polarization insensitive semiconductor traveling wave amplifier with low driving current," Electron. Lett., vol. 26, pp. 124-125, 1990.

[3] L. F. Tiemeijer, P. J. A. Thijs, T. van Dongen, J. J. M. Binsma, and E J. Jansen, "Polarization resolved, complete characterization of $1310 \mathrm{~nm}$ fiber pigtailed multiple-quantum-well optical amplifiers," J. Lightwave Technol., vol. 14, pp. 1524-1533, 1996.

[4] T. D. Visser, H. Blok, and D. Lenstra, "Modal analysis of a planar waveguide with gain and losses," IEEE J. Quantum Electron., vol. 31, pp. 1803-1810, 1995.

[5] T. D. Visser, B. Demeulenaere, J. Haes, D. Lenstra, R. Baets, and H. Blok, "Confinement and modal gain in dielectric waveguides," $J$. Lightwave Technol., vol. 14, pp. 885-887, 1996.

[6] J. Haes, B. Demeulenaere, T. D. Visser, D. Lenstra, H. Blok, and R. Baets, "How (not) to calculate the confinement factor for the modal gain of TM modes in amplifying waveguides," in Proc. ECOC'95, 1995, pp. 729-732, paper We.P. 31

[7] J. Haes, B. Demeulenaere, T. D. Visser, D. Lenstra, H. Blok, and R. Baets, "Difference between TE and TM modal gain in amplifying waveguides: Analysis and assessment of two perturbation approaches," Opt. Quantum Electron., vol. 29, pp. 263-273, 1997.

[8] J. Buus, "The effective index method and its application to semiconductor lasers,” IEEE J. Quantum Electron., vol. QE-18, pp. 1083-1089, 1982.

[9] H. Ghafouri-Shiraz, Fundamentals of Laser Diode Amplifiers. New York: Wiley, 1996, ch. 4.

[10] R. F. Harrington, Time-Harmonic Electromagnetic Fields. New York: McGraw-Hill, 1961, ch. 7.

[11] M. J. Adams, An Introduction to Optical Waveguides. New York, Wiley, 1981, sec. 2.3.5.

[12] Huang et al., "Analysis of the optical confinement factor in semiconductor lasers," Appl. Phys., vol. 79, pp. 3827-3830, 1996.

[13] D. Yevick, "A guide to electric field propagation techniques for guidedwave optics," Opt. Quantum Electron., vol. 26, pp. S185-S197, 1994.

[14] H. J. W. M. Hoekstra, "On beam propagation methods for modeling in integrated optics," Opt. Quantum Electron., vol. 29, pp. 157-171, 1997.

[15] A. T. de Hoop, Handbook of Radiation and Scattering of Waves. London, U.K.: Academic, 1995, ch. 21.

[16] J. D. Jackson, Classical Electrodynamics, 2nd ed. New York: Wiley, 1975 , ch. 6.

[17] C. Vassallo, "Circulair fourier analysis of full Maxwell equations for arbitrarily shaped dielectric waveguides-Application to gain factors of semiconductor laser waveguides," J. Lightwave Technol., vol. 8, pp. 1723-1729, 1990.

[18] H. Ghafouri-Shiraz and C. Y. J. Chu, "Analysis of waveguiding properties of travelling-wave semiconductor laser amplifiers using perturbation technique," Fiber and Integrated Opt., vol. 11, pp. 51-70, 1992.

[19] H. M. de Ruiter, "Limits on the propagation constants of planar optical waveguide modes," Appl. Opt., vol. 20, pp. 731-732, 1981.

T. D. Visser received the Ph.D. degree from the University of Amsterdam, Amsterdam, The Netherlands, in 1992.

$\mathrm{He}$ is now an Assistant Professor of Theoretical Physics at the Free University, Amsterdam. His research interests are semiconductor lasers and amplifiers and the theory of diffraction and scattering.

H. Blok (M'87) is a Professor of Electromagnetics at Delft University of Technology, Delft, The Netherlands. His research focuses on beam propagation methods, integrated optics, and inverse scattering theory.

B. Demeulenaere (S'90-M'93) has just finished his doctoral thesis at the University of Gent, Gent, Belgium. His research focused on wave propagation in vertical-cavity surface-emitting lasers and on the quantum electrodynamics of spontaneous emission in such structures.

D. Lenstra (M'97) is a Professor of Theoretical Physics and Dean of the Department of Physics and Astronomy at the Free University, Amsterdam, The Netherlands. He conducts research on various apsects of semiconductor lasers such as nonlinear dynamics, wave propagation, and quantum electrodynamics. 